\title{
MECHANICAL PROPERTIES AND ADVANCED SUBJECTS IN SHAPE MEMORY ALLOYS AND POLYMERS
}

\author{
Ryosuke Matsui, Kohei Takeda, Hisaaki Tobushi \\ Department of Mechanical Engineering, Aichi Institute of Technology, Toyota, Japan \\ e-mail:r_matsui@aitech.ac.jp; k-takeda@aitech.ac.jp; tobushi@aitech.ac.jp \\ ElzBieta A. Pieczyska \\ Institute of Fundamental Technological Research, Polish Academy of Sciences, Warsaw, Poland \\ e-mail: epiecz@ippt.pan.pl
}

\begin{abstract}
Advanced subjects in mechanical properties of shape memory alloys and polymers are discussed. In the subloop loading under a stress-controlled condition of the shape memory alloy, the transformation-induced stress relaxation appears due to variation in temperature. The enhancement of corrosion and corrosion fatigue life of the shape memory alloy is discussed. The development of a functionally-graded shape memory alloy and polymer is expected to obtain better performance. Three-way motion appears in the shape memory composite with the shape memory alloy and polymer.
\end{abstract}

Keywords: shape memory alloy, shape memory polymer, functionally-graded shape memory material, shape memory composite

\section{Introduction}

The development of shape memory alloys (SMA) has attracted high attention because of the unique properties of the shape memory effect (SME) and superelasticity (SE) appearance (Duerig et al., 1990; Funakubo, 1987; Lagoudas, 2008; Lexcellent, 2013; Otsuka and Wayman, 1998; Sun et al., 2017; Tobushi et al., 2013; Ziolkowski, 2015). If we use the SME and SE in practical applications, not only large recovery strain but also high recovery stress, energy storage and energy dissipation can be obtained. The main features of the SME and SE are induced due to martensitic transformation (MT). Since the deformation properties due to the MT depend on temperature, stress and thermomechanical hysteresis, they are, therefore, complex. They also depend on the loading rate. In the case of subloop loading, the deformation behaviors are quite different between strain- and stress-controlled loading conditions. The transformation-induced creep and stress relaxation appear in the subloop loading under the stress-controlled condition. The corrosion and corrosion fatigue properties are important in practical application of SMA elements.

The shape memory polymer (SMP) has also been developed (Hayashi, 1993; Huang et al., 2012; Tandon et al., 2016; Yahia, 2015). The main features of SMP appear due to the glass transition. Elastic modulus differs at temperatures above and below the glass transition temperature, and the rigidity of SMP elements, therefore, varies depending on the temperature change. Based on this property, the shape fixity and shape recovery can be used.

Although elastic modulus and yield stress are high at high temperatures and low at low temperatures in SMAs, they are high at low temperatures and low at high temperatures in SMPs. The dependence of rigidity on temperature is, therefore, quite different between the SMA and SMP. If composite materials with SMA and SMP are developed, new characteristics of the shape memory materials can be obtained. 
In order to obtain a better performance, the development of functionally-graded SMAs and SMPs is expected. The 3D-printing of SMPs is requested as a simple method to manufacture complex SMP elements.

In the present paper, advanced subjects in mechanical properties of SMA such as the deformation behavior subjected to the stress-controlled subloop loading and the corrosion fatigue properties of SMA are discussed. Next, the functionally-graded SMA and SMP are discussed. Following these subjects, the mechanical properties of the shape memory composite with SMA and SMP such as the characteristics of the three-way bending properties and the $3 \mathrm{D}$ printing of SMP will be discussed.

\section{Deformation and fatigue properties of SMAs}

\section{1. $\quad$ Stress relaxation in subloop loading}

SMA elements are subjected to variation in stress, strain and temperature with various ranges accompanying the MT in practical applications. The analysis in the subloop loading is therefore important. Although the return-point memory appears under a low strain rate in the subloop loading, it does not appear under the stress-controlled condition. In the case of the subloop loading under the stress-controlled condition, the transformation-induced creep and creep recovery appear under a constant stress, and the transformation-induced relaxation and stress recovery under a constant strain. It should be noticed that the stress-strain curve depends on the loading rate (Ikeda, 2015; Pieczyska et al., 2006; Yin et al., 2014). The transformation-induced stress relaxation in the subloop loading of the TiNi SMA under various loading conditions will be discussed in this Section.

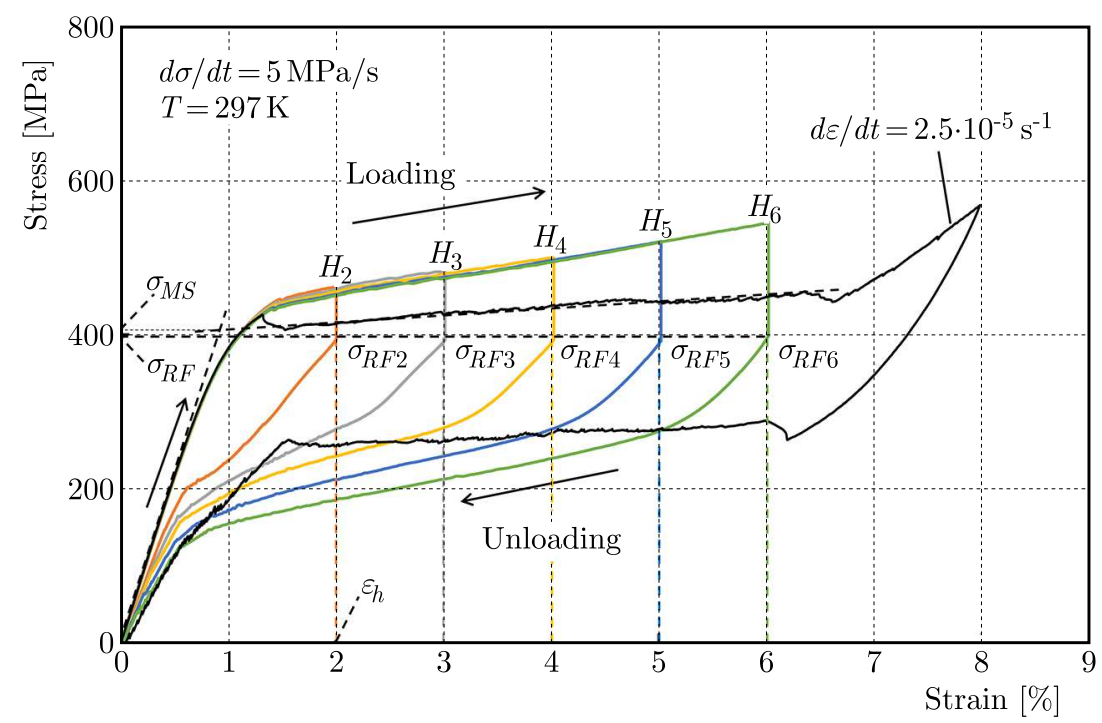

Fig. 1. Stress-strain curves of the TiNi SMA in the stress relaxation test with various holding strains and in the low strain rate $d \varepsilon / d t=2.5 \cdot 10^{-5} \mathrm{~s}^{-1}$

The stress-strain curves obtained in a stress relaxation test with various holding strains are shown in Fig. 1. The loading conditions of the stress relaxation tests in Fig. 1 were as follows. The load was applied at a stress rate $d \sigma / d t=5 \mathrm{MPa} / \mathrm{s}$ until a point $H_{2}\left(\right.$ or $H_{3}, H_{4}, H_{5}$ and $H_{6}$ ) at a strain $\varepsilon_{h}=2 \%$ (or $3 \%, 4 \%, 5 \%$ and $6 \%$ ) followed by holding the strain $\varepsilon_{h}$ constant till the decrease in stress finished and thereafter unloaded at a stress rate $d \sigma / d t=-5 \mathrm{MPa} / \mathrm{s}$. The stress-strain curve shown by a black line in Fig. 1 was obtained at a strain rate $d \varepsilon / d t=2.5 \cdot 10^{-5} \mathrm{~s}^{-1}$ during loading and unloading with the maximum strain $8 \%$. As can be seen in Fig. 1, in the strain 
holding process at $\varepsilon_{h}$ following the loading till the strain $\varepsilon_{h}$ at the stress rate $d \sigma / d t=5 \mathrm{MPa} / \mathrm{s}$, the stress decreases to $\sigma_{R F 2}$ (or $\sigma_{R F 3}, \sigma_{R F 4}, \sigma_{R F 5}$ and $\sigma_{R F 6}$ ), resulting in stress relaxation. The stress $\sigma_{R F 2}$ (or $\sigma_{R F 3}, \sigma_{R F 4}, \sigma_{R F 5}$ and $\sigma_{R F 6}$ ) at a point $\varepsilon_{h}$ after relaxation is almost the same as the stress of the MT start $\sigma_{M S}$ in the stress-strain curve at a strain rate $d \varepsilon / d t=2.5 \cdot 10^{-5} \mathrm{~s}^{-1}$, in which an increase in the stress is smaller than that at a stress rate $d \sigma / d t=-5 \mathrm{MPa} / \mathrm{s}$.

In the loading process at a constant stress rate $d \sigma / d t=5 \mathrm{MPa} / \mathrm{s}$, strain rate becomes high in the upper stress plateau region, and the heat is generated due to the exothermic MT, resulting in an increase in temperature of the specimen. In the strain holding stage from the point $H_{2}$ (or $H_{3}, H_{4}, H_{5}$ and $H_{6}$ ) to $\sigma_{R F 2}$ (or $\sigma_{R F 3}, \sigma_{R F 4}, \sigma_{R F 5}$ and $\sigma_{R F 6}$ ), the temperature decreases due to the heat transfer into the ambient air and the condition for the transformation to progress is satisfied, resulting in progress of the MT. As a result, stress relaxation occurs while holding the strain constant.

The relationship between the stress decrease $\Delta \sigma$ and temperature decrease $\Delta T$ during holding a constant strain in the stress relaxation tests with various conditions is shown in Fig. 2 . Temperature was measured by the infrared thermography. The forced convection was performed by air flow in order to observe the influence of cooling rate on stress relaxation. As can be seen, the stress decrease $\Delta \sigma$ is proportional to the temperature decrease $\Delta T$. The broken line in Fig. 2 is calculated by $\Delta \sigma=a \Delta T$ and shows a good overall match with the experimental results. The value of the coefficient $a$ is $13.2 \mathrm{MPa} / \mathrm{K}$.

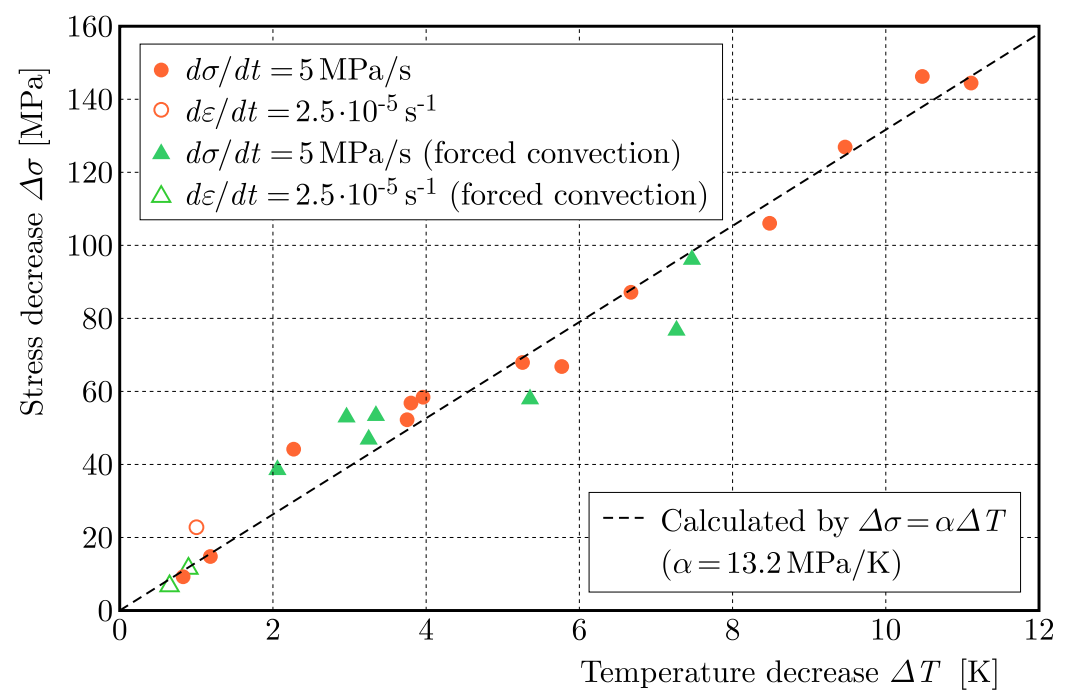

Fig. 2. Relationship between stress decrease and temperature decrease in the stress relaxation test with various conditions

\subsection{Corrosion and fatigue properties of SMA}

\subsubsection{Corrosion fatigue life}

The corrosion fatigue life is important in practical applications of SMAs. However, the report on the corrosion fatigue properties is little. The corrosion fatigue life of a TiNi SMA wire was investigated through a bending fatigue test.

The relationships between the strain amplitude $\varepsilon_{a}$ and the number of cycles to failure $N_{f}$ obtained by the rotating-bending fatigue test in the air and the $10 \%-\mathrm{NaCl}$ water solution are shown in Fig. 3 (Yamada and Matsui, 2016). The materials used in the experiment were TiNi SMA wires (Ti-49.7 at\% Ni) with a diameter of $0.7 \mathrm{~mm}$. The materials were heat-treated in an electrical furnace for $1 \mathrm{~h}$ at $673 \mathrm{~K}$. The materials were then allowed to cool inside the furnace. The fatigue life in $10 \%-\mathrm{NaCl}$ water solution (i.e. the corrosion fatigue life) is shorter than that in the air as shown in Fig. 3. Accordingly, engineers have to be careful with fatigue life of SMA 
devices, particularly, when used in corrosive environment (i.e. in human body, seawater, etc.). In order to enhance the corrosion fatigue life, we have developed a thermal treatment to generate a strong and homogeneous passive layer on the surface of TiNi SMAs. This subject will be discussed in the next Section.

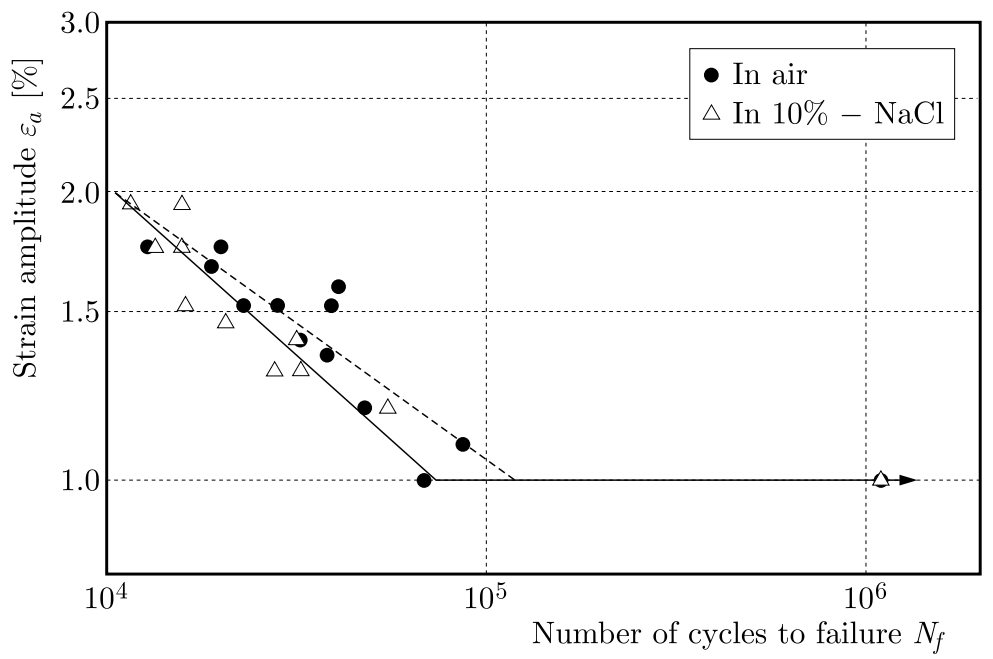

Fig. 3. Fatigue life curves for TiNi shape memory alloys in the air and $10 \%-\mathrm{NaCl}$ water solution

The enhancement of fatigue life can also be achieved by the surface treatment of materials through the ultrasonic shot peening (USP) and the nitrogen ion implantation (NII). The influence of NII, USP and thermal treatment conditions on the corrosion fatigue life of an SME tape and an SE tape is the future subject.

\subsubsection{Corrosion resistance}

In order to promote the application of SMAs into devices used in corrosive environment, we have developed a procedure to generate a passive layer on TiNi SMAs. The proposal process called the thermal nitridation (TN) treatment to generate thin and homogeneous passive layers contains heat treatment of a mechanically-polished SMA wire at temperature of $673 \mathrm{~K}$ for $3.6 \mathrm{ks}$ in a furnace filled with pure nitrogen gas.

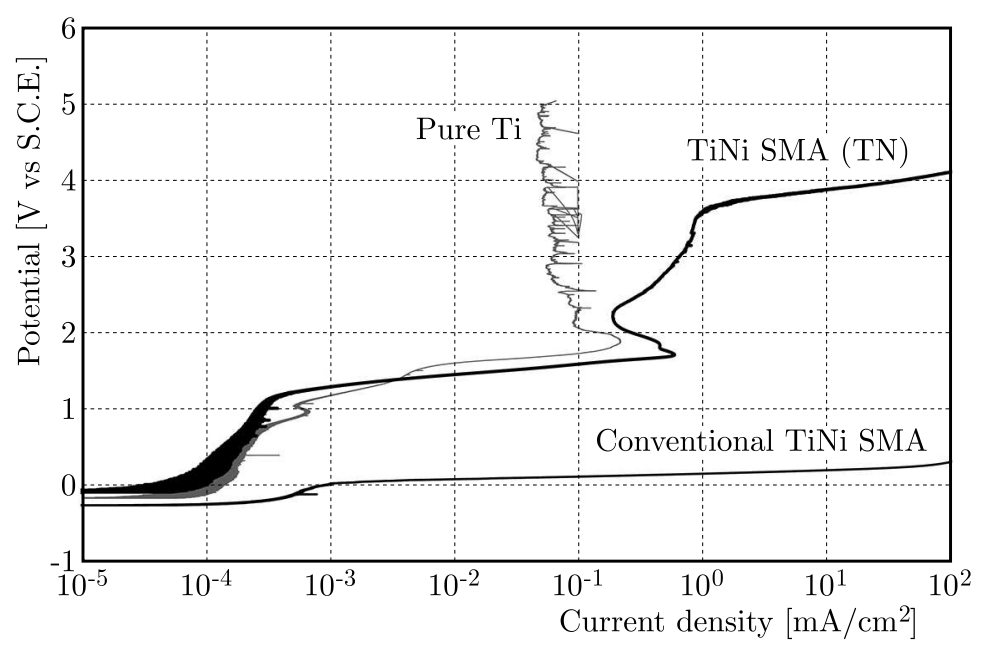

Fig. 4. Anodic polarization curves for TiNi SMA (TN), conventional TiNi SMA and pure Ti

Figure 4 shows anodic polarization curves for TiNi SMA with the passive layer generated by TN process, a conventional TiNi SMA with a thick oxide layer and a pure Ti in a $3 \%-\mathrm{NaCl}$ water 
solution. The results reveal that corrosion resistance of the TN-treated TiNi SMA is much higher than that of the conventional TiNi SMAs and is almost the same as that of pure Ti, which is the most common material as a biomaterial, with current density of up to above $1 \cdot 10^{-2} \mathrm{~mA} / \mathrm{cm}^{2}$. From the energy dispersive X-ray spectrometry analysis and other microscopic investigations, we found that a thin titanium-nitride layer with a thickness of tens of nanometers is generated on the TN-treated TiNi SMA.

Since the corrosion fatigue life for the TN-treated TiNi SMA is one of the important properties to design devices, we are now investing in clarifying the characteristics of corrosion fatigue life from a fatigue test in the $10 \%-\mathrm{NaCl}$ water solution.

\section{Functionally-graded shape memory alloy and shape memory polymer}

\subsection{Functionally-graded shape memory alloy}

In order to develop a more advanced actuator, such as a self-stroke controlling device depending on the ambient temperature, a TiNi SMA having a functionally-graded property of the transformation temperatures will be a major candidate material for the element. If the functionally-graded shape memory alloy (FGSMA) coil is employed to an actuator, the shape-recoverable region exceeding the austenitic transformation finish temperature $A_{f}$ will change continuously, resulting in length of the coil extending or shortening without a bias element, depending only on its temperature.

Figure 5 shows a demonstration of movement of an FGSMA coil having different transformation temperatures $A_{f} ; A_{f 1}=293 \mathrm{~K}, A_{f 2}=318 \mathrm{~K}$ and $A_{f 3}=338 \mathrm{~K}$. The coil subjected to tensile load along its axial direction and then it was unloaded at temperature $T=298 \mathrm{~K}$ as shown in Figs. 5b and 5c. In this state, a part of the coil, which had the transformation temperature $A_{f 1}$, recovered its original shape without heating due to superelasticity. The coil was subsequently heated up to $T=328$ and $348 \mathrm{~K}$, the shape recoverable region extended and length of the coil became shorter as shown in Figs. 5d and 5e.

(a)

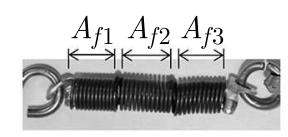

(b)

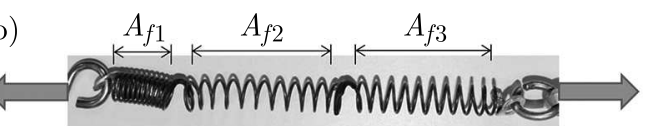

(c)

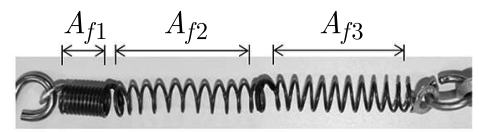

(d)

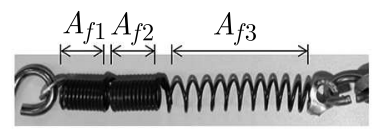

(e)

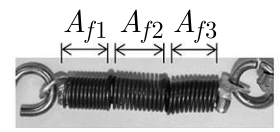

Fig. 5. Movement of the SMA coil having different transformation temperatures; $A_{f 1}=293 \mathrm{~K}$, $A_{f 2}=318 \mathrm{~K}$ and $A_{f 3}=338 \mathrm{~K}$ : (a) initial state, (b) loading at $T=298 \mathrm{~K}\left(A_{f 1}<T<A_{f 2}<A_{f 3}\right)$,

(c) unloading at $T=298 \mathrm{~K}\left(A_{f 1}<T<A_{f 2}<A_{f 3}\right)$, (d) heating up to $T=328 \mathrm{~K}$ $\left(A_{f 1}<A_{f 2}<T<A_{f 3}\right)$, (e) heating up to $T=348 \mathrm{~K}\left(A_{f 1}<A_{f 2}<A_{f 3}<T\right)$ 
As an example of manufacturing the functionally-graded shape memory alloy (FGSMA), we have developed a new fabrication process that combines powder metallurgy and hot extrusion to obtain an FGSMA wire in which the transformation temperature varies from high to low along the wire axis depending on gradually changing composition of Ti and Ni. First, a multilayered TiNi green compact in which the Ti-Ni compositions varied layer by layer was sintered using a spark plasma sintering process and then the compact was hot extruded into a wire. We used a characteristic that the phase transformation temperature of TiNi SMA changed depending on the composition of $\mathrm{Ti}$ and Ni (Duerig et al., 1990; Funakubo, 1987; Otsuka and Wayman, 1998).

Figure 6 shows the stress-local strain curves at three points of the hot extruded wire with gradually changing composition of $\mathrm{Ti}$ and $\mathrm{Ni}$. The wire shows the $\mathrm{SE}$ at the position that corresponds to a Ni content of 51.0 at\% and the SME at the position of 50.4 at\% Ni. These differences appear to be based on the different MT temperatures at each position.

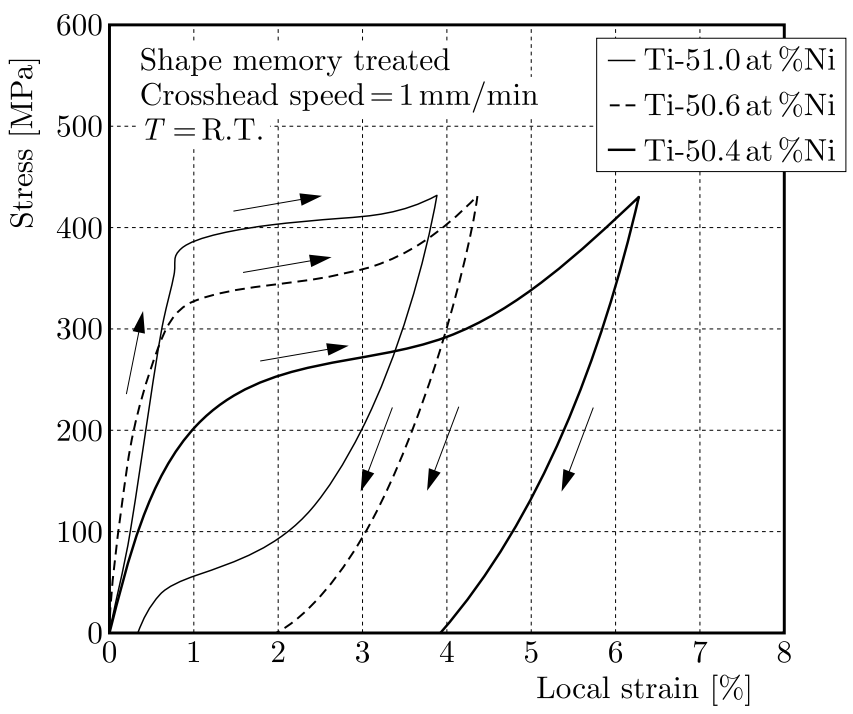

Fig. 6. Stress-local strain curves of FGSMA wire with gradually changing composition

If the FGSMA wire or tape is developed, the temperature-dependent continuous actuation or the multi-way actuation can be obtained by using only one SMA coil or tape, respectively.

\subsection{Functionally-graded shape memory polymer}

The relationship between force and depth of the functionally-graded shape memory polymer (FGSMP) board in the indentation test is similar to that of the finger (Takeda et al., 2016). The deformation properties of the body differ depending on the region. The FGSMP board corresponding to each region can be developed by a combination of the sheet and foam with appropriate thickness, glass transition temperature and their arrangement. The FGSMP board can therefore be applied to the elements coming into contact with the body as a nursing-care robot in the medical actuators. The basic deformation property of the FGSMP foam will be discussed in this Section.

A polyurethane-SMP foam has been used to fabricate FGSMP foams. The SMP foams having different glass transition temperatures $T_{g}\left(T_{g}=298 \mathrm{~K}\right.$ and $\left.321 \mathrm{~K}\right)$ and density $\rho\left(\rho=43 \mathrm{~kg} / \mathrm{m}^{3}\right.$ and $70 \mathrm{~kg} / \mathrm{m}^{3}$ ) were laminated and the FGSMP was fabricated. Thickness of each SMP foam was $10 \mathrm{~mm}$. The structure of an FGSMP foam is shown in Fig. 7. Two SMP foams with $T_{g}=298 \mathrm{~K}$ and $\rho=43 \mathrm{~kg} / \mathrm{m}^{3}$ were arranged in the upper part, and those with $T_{g}=321 \mathrm{~K}$ and $\rho=70 \mathrm{~kg} / \mathrm{m}^{3}$ in the lower part. The adhesive used to laminate the foam was DW246W produced by SMP Technologies Inc. 


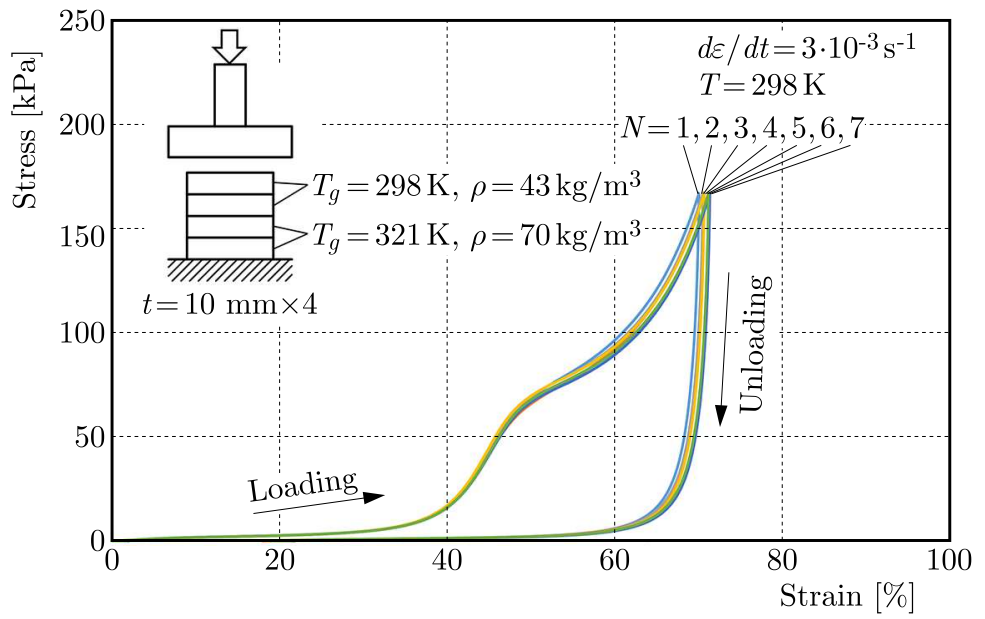

Fig. 7. Stress-strain curves of FGSMP foam in the thermomechanical compression test

The thermomechanical cyclic compression test was performed on the FGSMP foam. At first, it was compressed at a strain rate of $3 \cdot 10^{-3} \mathrm{~s}^{-1}$ in the loading and unloading processes at the room temperature $T=298 \mathrm{~K}$. Thereafter, the FGSMP foam was heated up to $373 \mathrm{~K}$ for 5 minutes in the furnace to recover the residual strain followed by cooling down to the room temperature. These processes were repeated in 7 cycles. The relationship between stress and strain obtained in the cyclic compression test is shown in Fig. 7. As can be seen, the stress increases slightly at the initial stage due to deformation of the SMP foam with $T_{g}=298 \mathrm{~K}$ and $\rho=43 \mathrm{~kg} / \mathrm{m}^{3}$. Then, the stress increases rapidly around a strain of $45 \%$ due to completion of local deformation of the upper SMP foam. Thereafter, the SMP foam with $T_{g}=321 \mathrm{~K}$ and $\rho=70 \mathrm{~kg} / \mathrm{m}^{3}$ was deformed accompanying the stress plateau starting from about $70 \mathrm{kPa}$, resulting in two steps of deformation in the loading process. After unloading, the residual strain of $19 \%$ appears under no-load due to the irrecoverable strain of the SMP foam with $T_{g}=321 \mathrm{~K}$. This residual strain can be recovered almost to $0 \%$ if the FGSMP is heated above $T_{g}=321 \mathrm{~K}$ under no-load. The deformation behavior is almost the same in each cycle.

\section{Shape memory composite with SMA and SMP}

The structure and a photograph of the fabricated shape memory composite (SMC) belt is shown in Fig. 8. As can be seen in Fig. 8a, the 3D-printed SMP sheet, SME wire and SE wire were laminated. Then the laminated material was set in the mold for heat-treating of the SMC belt. The glass transition temperature $\left(T_{g}=328 \mathrm{~K}\right)$ of the SMP sheet was above the both phase transformation temperatures $A_{f}$ of the SME wire $\left(A_{f 2}=316 \mathrm{~K}\right)$ and SE wire $\left(A_{f 1}=306 \mathrm{~K}\right)$ of TiNi alloy. The SME and SE wires were arranged facing in the opposite directions for the memorized U-shape. The SME and SE wires were sandwiched between two 3D-printed SMP sheets from the upper and lower sides. The SMC belt shown in Fig. 8b can be fabricated without bubbles and gaps by using the 3D-printed SMP sheet.

The relationship between the recovery force and temperature of the SMC belt operated by joule heating of both wires is shown in Fig. 9. As can be seen, during heating by joule heat, the recovery force decreases from point (I) to (II) due to the recovery force of SE wire. Then, the recovery force of the SMC belt increases from point (II) to (III) since the recovery force of the SME wire appears. During cooling by the ambient air, the recovery force of the SMC belt decreases from point (III) to (IV) due to decreasing in the recovery force of the SME wire. Then the force of the SMC belt becomes constant since the SMP sheet becomes harder below $T_{g}$. The deformation behavior of the SMC belt is almost the same in the cyclic heating and cooling 
(a) 3D-printed SMP sheet $\left(t=0.2 \mathrm{~mm}, T_{g}=328 \mathrm{~K}\right)$

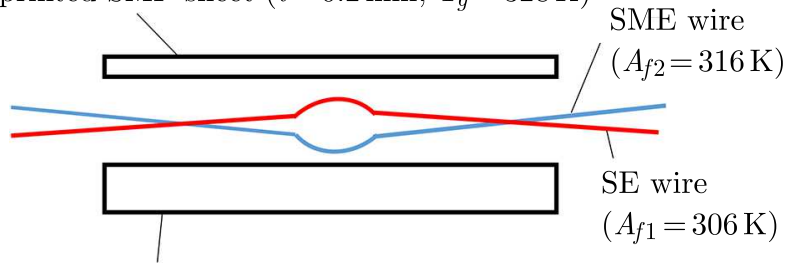

3D-printed SMP sheet $\left(t=0.6 \mathrm{~mm}, T_{g}=328 \mathrm{~K}\right)$

(b)

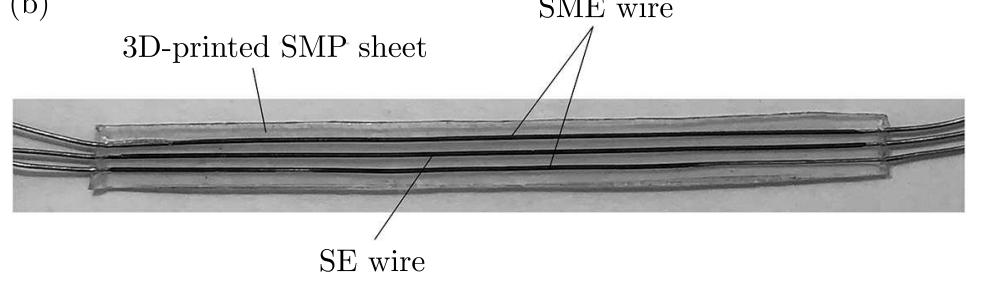

Fig. 8. Structure (a) and photograph (b) of the SMC belt

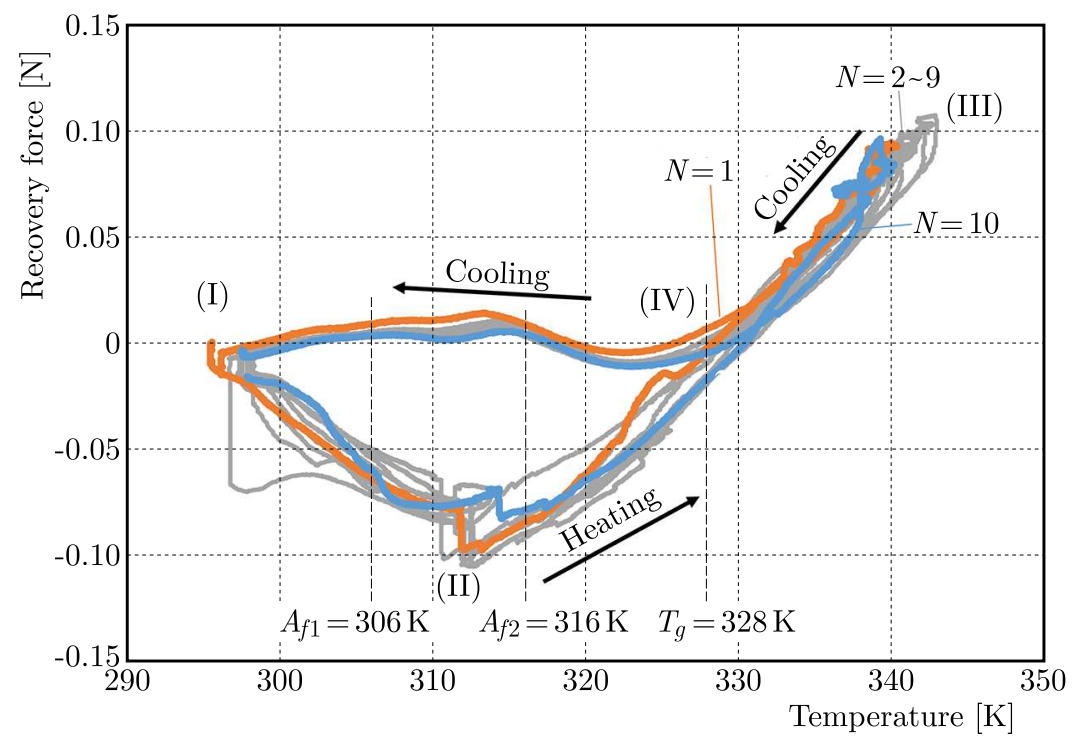

Fig. 9. Variation in the recovery force of the SMC belt during cyclic heating and cooling

processes. The reciprocating three-way behavior of the recovery force can be obtained by a simple SMC structure.

\section{3D-Printed shape memory polymer}

Recently, the 3D printer which can make products in a short time without cutting or casting has been attracted worldwide attention. If we use the $3 \mathrm{D}$ printer, it is possible that a customized product which is well suited to the individual is fabricated with a low cost and in a short time. If we make a product with SMP using a 3D printer, the new device which is well suited to the individual complex shape can be developed without using expensive metal molds.

The material used to fabricate a 3D-printed SMC belt was a SMP filament which was newly developed by SMP Technologies Inc. and KYORAKU Co., Ltd. The 3D-printed SMP belt was fabricated by a fused deposition modeling (FDM) 3D printer. The nozzle movement during printing the belt is shown in Fig. 10. The pattern of movement of the nozzle during printing was 
$0^{\circ}$ angle with respect to the longitudinal direction of the belt. The thickness of each layer in the printing process was $0.2 \mathrm{~mm}$. The total layers were two and thickness of the belt was $0.4 \mathrm{~mm}$.

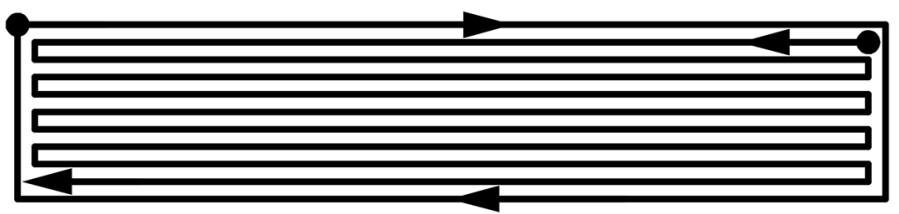

Fig. 10. Nozzle movement during fabricating an SMP belt in 3D-printing

Photographs of motion of the 3D-printed SMP belt for shape fixity and shape recovery are shown in Fig. 11. As can be seen, (1) the 3D-printed SMP belt was deformed at a temperature above $T_{g}$. Then, (2) the deformed shape was maintained without a force at temperatures below $T_{g}$. Next, (3) the original shape of the belt was recovered by heating above $T_{g}$. The shape fixity and shape recovery properties are obtained in the 3D-printed SMP as same as the SMP made by the general method. The thermomechanical properties of the 3D-printed SMP depend on the nozzle temperature, table temperature, printing rate and pattern of each layer (Takeda et al., 2017). These points are the future subjects.

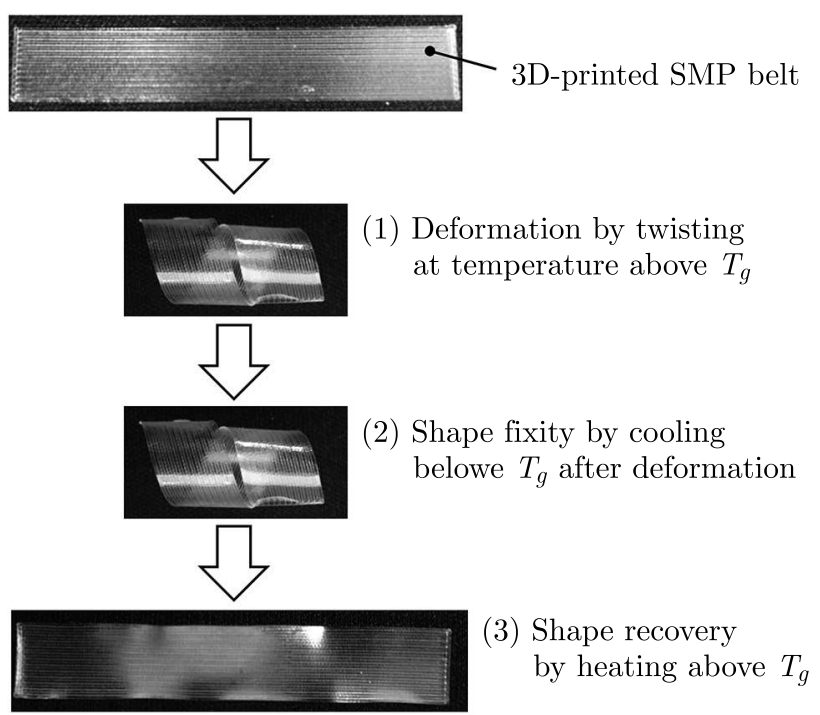

Fig. 11. Photographs of shape fixity and shape recovery of the 3D-printed SMP belt

\section{Conclusions}

Mechanical properties and advanced subjects in shape memory alloys and polymers have been discussed. The results obtained are summarized as follows.

- The shape memory effect and superelasticity of shape memory alloys depend on thermomechanical loading conditions. The transformation-induced stress relaxation appears in the subloop loading under the stress-controlled condition. The stress decrease is proportional to the temperature decrease during holding the strain constant.

- The thin titanium-nitride layer with thickness of tens of nanometers is generated on TNtreated TiNi SMA. The enhancement of corrosion fatigue life of the shape memory alloy is expected by thermal nitridation treatment.

- The functionally-graded shape memory alloy and polymer with various compositions and phase transformation temperatures are fabricated to obtain better performance of shape memory elements. 
- Three-way motion can be obtained by the composite with shape-memory alloys and polymers having various phase transformation temperatures.

- The shape fixity and recovery properties can be obtained by a 3D-printed shape memory polymer element.

Acknowledgement

The authors are grateful for the support of JSPS KAKENHI Grant No. JP15K21477 and the National Science Centre (NCN), Poland, under Grant 2014/13/B/ST8/04280.

\section{References}

1. Duerig T.W., Melton, K.N., Stokel D., Wayman C.M., edit., 1990, Engineering Aspects of Shape Memory Alloy, Butterworth-Heinemann, Oxford

2. Funakubo H., Edit., 1987, Shape Memory Alloys, Gordon and Breach Science Pub., New York

3. HAYASHi S., 1993, Properties and applications of polyurethane series shape memory polymer, [In:] International Progress in Urethanes, 6, 90-115

4. Huang W.M., Yang B., Fu Y.Q., 2012, Polyurethane Shape Memory Polymers, CRC Press, Boca Raton

5. Ikeda T., 2015, Analytical investigation of strain loading frequency effect on stress-straintemperature relationship of shape-memory alloy, Archives of Mechanics, 67, 4, 275-291

6. Lagoudas D.C., Edit., 2008, Shape Memory Alloys, Springer, New York

7. Lexcellent C., 2013, Shape-Memory Alloys Handbook, John Wiley \& Sons, Hoboken

8. Otsuka K., Wayman C.M., Edit., 1998, Shape Memory Materials, Cambridge University Press, Cambridge

9. Pieczyska E.A., Tobushi H., Gadaj S.P., Nowacki W.K., 2006, Superelastic deformation behaviors based on phase transformation bands in TiNi shape memory alloy, Materials Transactions, $\mathbf{4 7}, 3,670-676$

10. Sun Q.P., Matsui R., Takeda K., Pieczyska E., edit., 2017, Advances in Shape Memory Materials, Cham, Springer

11. Takeda K., Hayashi S., UeKi K., 2017, Deformation properties of 3D printed shape memory polymer, Key Engineering Materials, 725, 378-382

12. Takeda K., Matsui R., Tobushi H., Hayashi S., 2016, Functionally-graded shape memory board, Mechanical Engineering Journal, 3, 6, 1-9, DOI: 10.1289/mej.16-00157

13. Tandon G.P., McClung A.J.W., Baur J.W., Edit., 2016, Shape Memory Polymers for Aerospace Applications, DEStech Publications, Inc., Lancaster

14. Tobushi H., Matsui R., Takeda K., Pieczyska E.A., 2013, Mechanical Properties of Shape Memory Materials, Nova Science Pub., New York

15. Yahia L.H., Edit, 2015, Shape Memory Polymers for Biomedical Applications, Elsevier, Amsterdam

16. YAmAda K., Matsui R., 2016, Improvement of corrosion fatigue strength for TiNi shape memory alloy, Key Engineering Materials, 725, 389-393

17. Yin H., He Y.J., Sun Q.P., 2014, Effect of deformation frequency on temperature and stress oscillation in cyclic phase transition of NiTi shape memory alloy, Journal of the Mechanics and Physics of Solids, 67, 100-128

18. Ziolkowski A., 2015, Pseudoelasticity of Shape Memory Alloys, Butterworth-Heinemann, Oxford 